Measuring the speed of electromagnetic waves using the cross correlation function of broadband noise at the ends of a transmission line

Alberto T. Pérez

Citation: American Journal of Physics 79, 1042 (2011); doi: 10.1119/1.3607431

View online: http://dx.doi.org/10.1119/1.3607431

View Table of Contents: http://aapt.scitation.org/toc/ajp/79/10

Published by the American Association of Physics Teachers

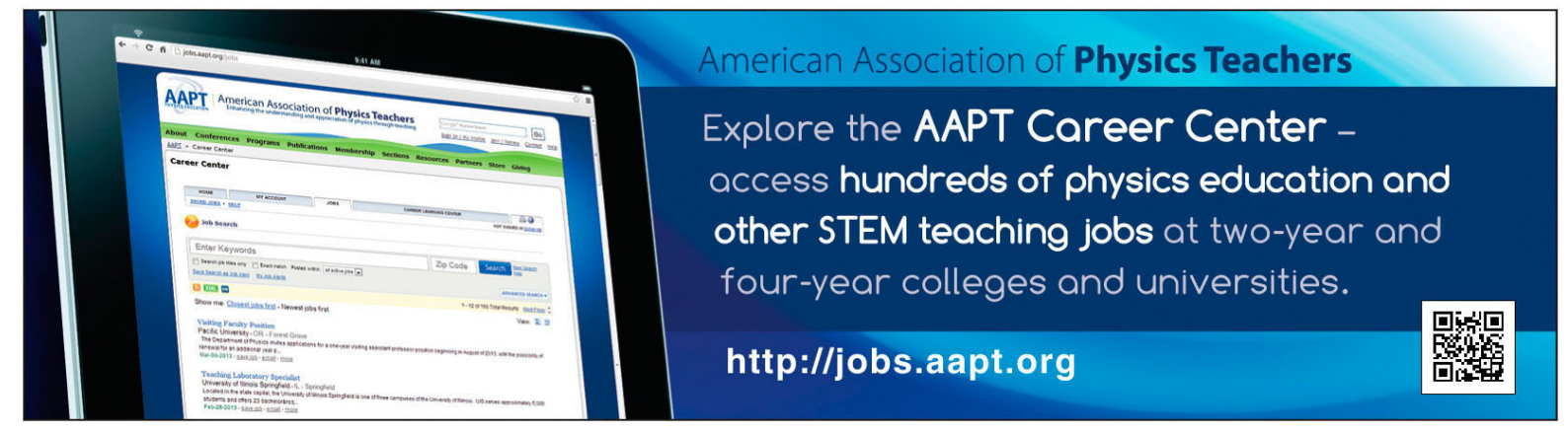




\title{
Measuring the speed of electromagnetic waves using the cross correlation function of broadband noise at the ends of a transmission line
}

\author{
Alberto T. Pérez ${ }^{\text {a) }}$ \\ Department of Electronics and Electromagnetism, University of Seville, 41012 Seville, Spain
}

(Received 13 January 2011; accepted 9 June 2011)

\begin{abstract}
When one end of a transmission line is connected to a broadband noise generator, sharp peaks are visible in the cross-correlation function of the signals at both ends of the line. The speed of the electromagnetic waves can be deduced from the time when the peaks appear. The method is suitable for introducing the concepts of cross-correlation functions and noise analysis in an undergraduate physics laboratory. (c) 2011 American Association of Physics Teachers.
\end{abstract}

[DOI: 10.1119/1.3607431]

Every signal is noise until we understand its structure. For example, the random walk of microscopic particles caused by thermal fluctuations may be used to evaluate Boltzmann's constant. ${ }^{1}$ Shot noise in electronic devices is a consequence of the discrete nature of the electron charge, ${ }^{2}$ and let us determine the charge of quasi-particles. ${ }^{3}$ Voltage fluctuations in a resistor are also related to thermal fluctuations and can be analyzed to investigate non-equilibrium thermodynamics. ${ }^{4}$

Autocorrelation functions and cross-correlation functions often play a central role in the statistical analysis of noisy signals. They are the basis of homodyne and heterodyne detection in photon correlation spectroscopy ${ }^{5}$ and similar techniques. The computation of correlation functions of ambient noise is being used to deduce the properties of the media in which waves propagate. $^{6}$ These studies may involve seismic waves, elastic waves, or other similar disturbances.

The method I discuss in this paper was used by Krafmakher ${ }^{7}$ to measure the speed of sound in air. Kraftmakher generated acoustic noise by connecting a loudspeaker to a noise voltage source and registered the signals detected by two microphones $90 \mathrm{~cm}$ apart. The cross-correlation function has a peak at the time needed for sound to cover the distance between the sensors. A similar measure can be done in a transmission line and leads to a determination of the speed of electromagnetic waves.

Watson $^{8}$ and Mak $^{9}$ discuss some of the concepts and applications of transmission lines. Some experiments are possible with a relative simple arrangement, including the determination of the speed of electromagnetic waves from the time required for a voltage pulse to cover the length of the line and a measurement of the characteristic impedance of the line by observing the reflections at the end of the line for different load resistors.

The same experimental set-up demonstrates the limit of validity of circuit theory, depending on the size of the circuit and the time scales involved. With the end of the line open, the transmission line behaves like a capacitor. If the driving resistor is such that the value $R C$ is much greater than $d / v(R$ is the value of the driving resistor, $C$ is the capacitance of the line, $d$ is its length, and $v$ is the speed of electromagnetic waves in the line), then the application of a step voltage to the line results in smooth charging as seen in a $R C$ circuit. If $R C$ is the same order of magnitude as $d / v$, then the charging proceeds in discrete steps, thus revealing the finite speed of propagation of the signals, and the failure of the circuit approximation.

In this paper, I present a simple method for measuring the speed of electromagnetic waves through a conventional transmission line by using the correlation of noisy signals at the ends of the line. The experimental set-up is suitable for an undergraduate physics laboratory and involves a piece of television coaxial cable. I use a digital oscilloscope, a programmable function generator, and a computer for further processing. A sufficiently fast data-acquisition-system is an alternative to the oscilloscope. The experiment introduces students to the use of cross-correlation functions.

The idea of the method is that part of the noise measured at both ends of a long transmission line results from the propagation of the same noisy signal through the line. Although both signals appear random, one signal is partly a time delayed copy. Therefore, the time cross-correlation function of both signals should have a maximum at the time corresponding to the transit of the electromagnetic waves along the line.

Figure 1 is a schematic representation of the experimental set-up. I used a $200 \mathrm{~m}$ long $75 \Omega$ coaxial cable (CLC75). The voltages at both ends of the line are measured with a digital dual trace oscilloscope. I used a Tektronix TDS 1012B, which has a maximum acquisition rate of $1 \mathrm{Gs} / \mathrm{s}$. This model has a USB port that can store the acquired signals in flash memory. The signals are transferred to a computer where the cross-correlation functions are computed.

I performed a preliminary test of the procedure by applying a square wave to one end of the transmission line. The square wave was provided by a conventional function generator. To obtain a clean signal at both ends of the line, the generator and the load were matched to the line. A load resistance of $75 \Omega$ was connected to the output of the line, and a series resistance of $75 \Omega$ (the internal resistance of the generator included) was connected to the input. In this way, the coefficients of reflection at both ends of the line are zero, and no reflected pulses are observed.

Figure 2(a) shows the reading of the two oscilloscope channels when the transmission line is fed with a square wave signal of $375 \mathrm{kHz}$. The line corresponding to channel 1 shows the voltage at the entrance. The channel 2 reading corresponds to the voltage at the exit of the line. The voltage at the exit is a delayed copy of the voltage at the entrance. The signal is attenuated due to line losses. The time difference between the two signals is $0.816 \pm 0.006 \mu \mathrm{s}$, which corresponds to the time of transit of electromagnetic waves along the line. Because the line is $200 \mathrm{~m}$ long, we deduce that the speed of electromagnetic waves in the line is $\approx 2.45 \times 10^{8} \mathrm{~m} / \mathrm{s}$.

To asses the synchronicity between the two channels I measured the signal at the same end with both oscilloscope probes. No time difference greater than the sample time was 


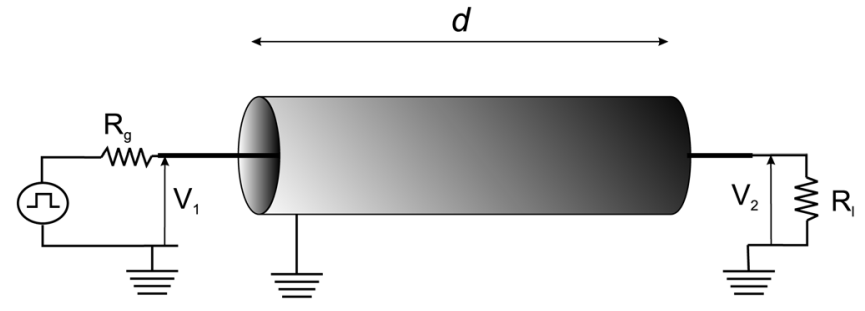

Fig. 1. Schematic representation of the transmission line with the function generator, the generator resistance, the loading resistance, and the oscilloscope probes.

found. ${ }^{10}$ In all the measurements discussed in this paper, the sampling time is in the range 1 to $4 \mathrm{~ns}$.

Once the signals were acquired I computed the autocorrelation and cross-correlation functions using MATLAB. The function xcorr of MATLAB computes the cross-correlation function of two arrays $x$ and $y$ according to the expression

$$
\begin{aligned}
& c(m)=R_{x y}(m-N) \\
& R_{x y}(m)= \begin{cases}\frac{1}{N-|m|} \sum_{n=0}^{N-m-1} x_{n+m} y_{n} & (m \geq 0), \\
R_{x y}(-m) & (m<0)\end{cases}
\end{aligned}
$$

where the index $m$ runs from 1 to $2 N-1$, with $N$ equal to the dimension of the arrays [ $R$ is an auxiliary quantity].

Figures 2(b) and 2(c) show the autocorrelation functions of the signals at the input and output of the line. The delay time in the horizontal axis is obtained multiplying the index $m$ by the sampling time. As expected, both functions are triangle wave-type with maxima at the origin. Figure $2(d)$ is a plot of the cross-correlation function of the signals displayed in Fig. 2(a).

The cross-correlation function of two delayed squarewave signals is a triangle wave function with the maximum at the time lag between the signals. Figure 2(d) confirms these expectations. The maximum of the cross-correlation function is at $0.82 \mu \mathrm{s}$, demonstrating that the determination of the cross-correlation functions of signals at both ends of the line serves as a way to evaluate the speed of electromagnetic waves.

Most interesting is the case when there is no external driving and no load. With both ends of the line open, I measured the voltage drop at each end. In this case, the voltage drop is caused by stray electromagnetic fields. Although there is some indication of a correlation at the expected time, the cross-correlation function of the signals at the ends of the line remains noisy, because it contains other correlations in addition to the one related to the propagation of signals along the line.

Stray electromagnetic fields are ubiquitous, but their magnitude is space- and time-dependent. The ideal for a cross correlation measurement is broadband noise. Stray electromagnetic fields often have a main frequency and its harmonics. Some electronic devices generate electromagnetic fields in the $\mathrm{MHz}$ range. The function generator I used to generate the signal shown in Fig. 2 includes an internal clock that
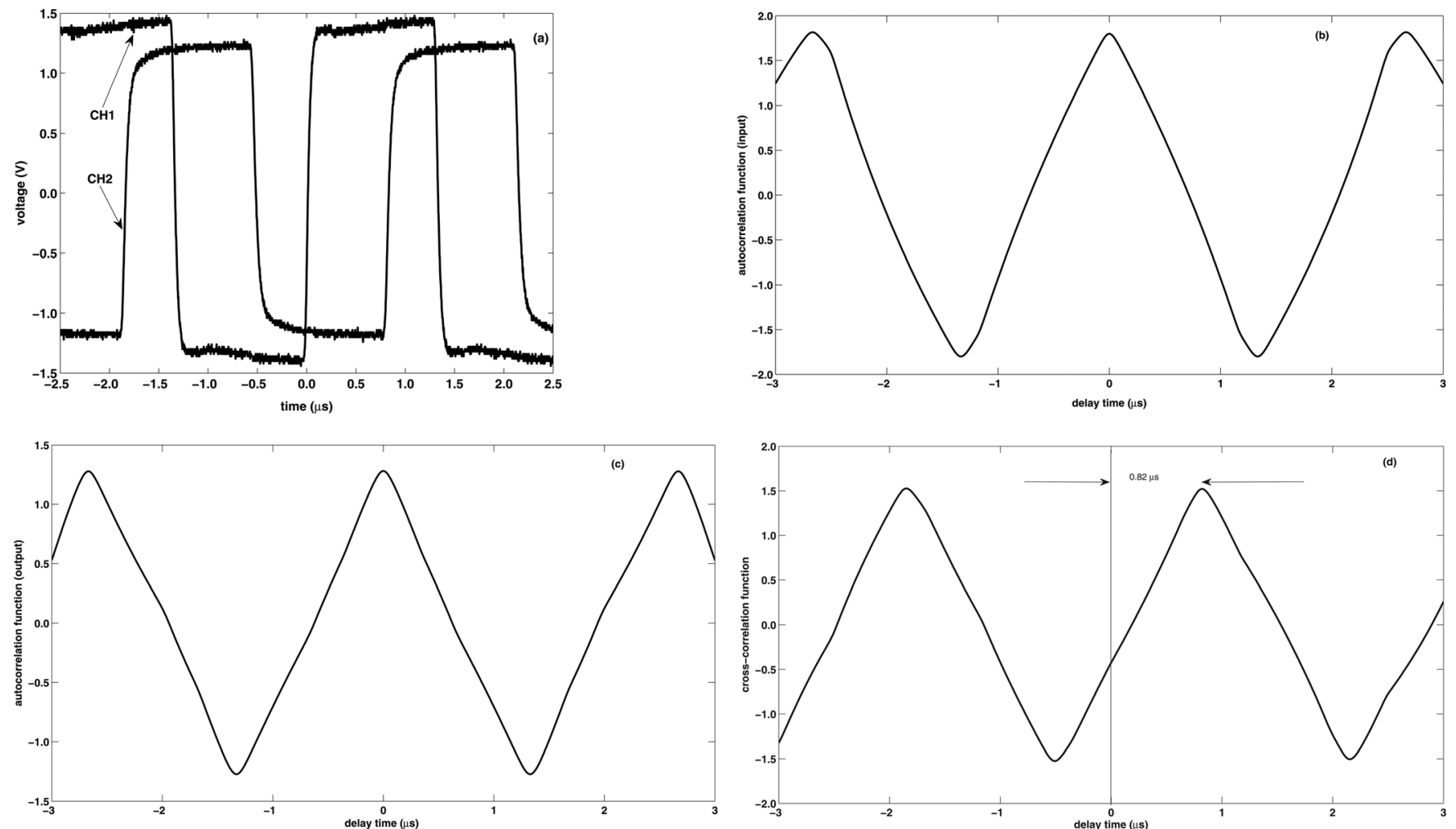

Fig. 2. Line response to a square signal: (a) Reading of the two channels of the oscilloscope when a square signal is applied to the transmission line. The reading of the second channel lags behind the first channel by a time that is equal to the transit of the electromagnetic pulse along the line. In this arrangement the value of the delay is $0.816 \mu \mathrm{s}$. The driving and load resistors are chosen to match the line impedance. (b) Autocorrelation function of the signal at the input of the line when a square signal is applied to it. This function is computed using Eq. (1) with $x$ and $y$ equal to the measured voltage waveform. (c) Autocorrelation function of the signal at the output of the line when a square signal is applied to it. (d) Cross-correlation function of the two signals. This function is computed using Eq. (1) with $x$ equal to the measured voltage waveform at the output and $y$ equal to the voltage waveform at the input. The maximum at $0.82 \mu$ s corresponds to the time of transit of the electromagnetic pulse along the line. 
produces a $10 \mathrm{MHz}$ signal. This signal is clearly detected near the apparatus and, because it gives several correlation peaks at times similar to the one to be measured, it is not useful for the purposes of this paper. Another problem arises if the signal level is not strong enough to excite measurable correlated voltages at the ends of the line. In that case, a long cable or a large conductor connected to the entrance of the line may help.

The use of cross-correlation functions to measure the velocity of electromagnetic signals is best illustrated by exciting the line with a broadband noise generator. I used a TTi TGA1244 (Ref. 11) arbitrary waveform generator to excite the transmission line with a noisy signal. The waveform was generated with MATLAB as a random vector of 1024 points. This waveform was emitted by the generator at a sample frequency of $20 \mathrm{MHz}$. The resulting signal presents a quasi-flat spectrum between $100 \mathrm{kHz}$ and $10 \mathrm{MHz}$. Figure 3(a) shows the signals at both ends of the transmission line. The reading corresponding to channel 2 has been displaced upward by 20 $\mathrm{V}$, to make both signals distinguishable. The function generator has an internal resistance of $50 \Omega$. I directly connected the output of the generator to the input of the line. The other end of the line was open.

The autocorrelation function for the signal at the input of the line in Fig. 3(b) shows two peaks at $\pm 1.62 \mu \mathrm{s}$. This time is twice the time of transit of the pulses along the line. The
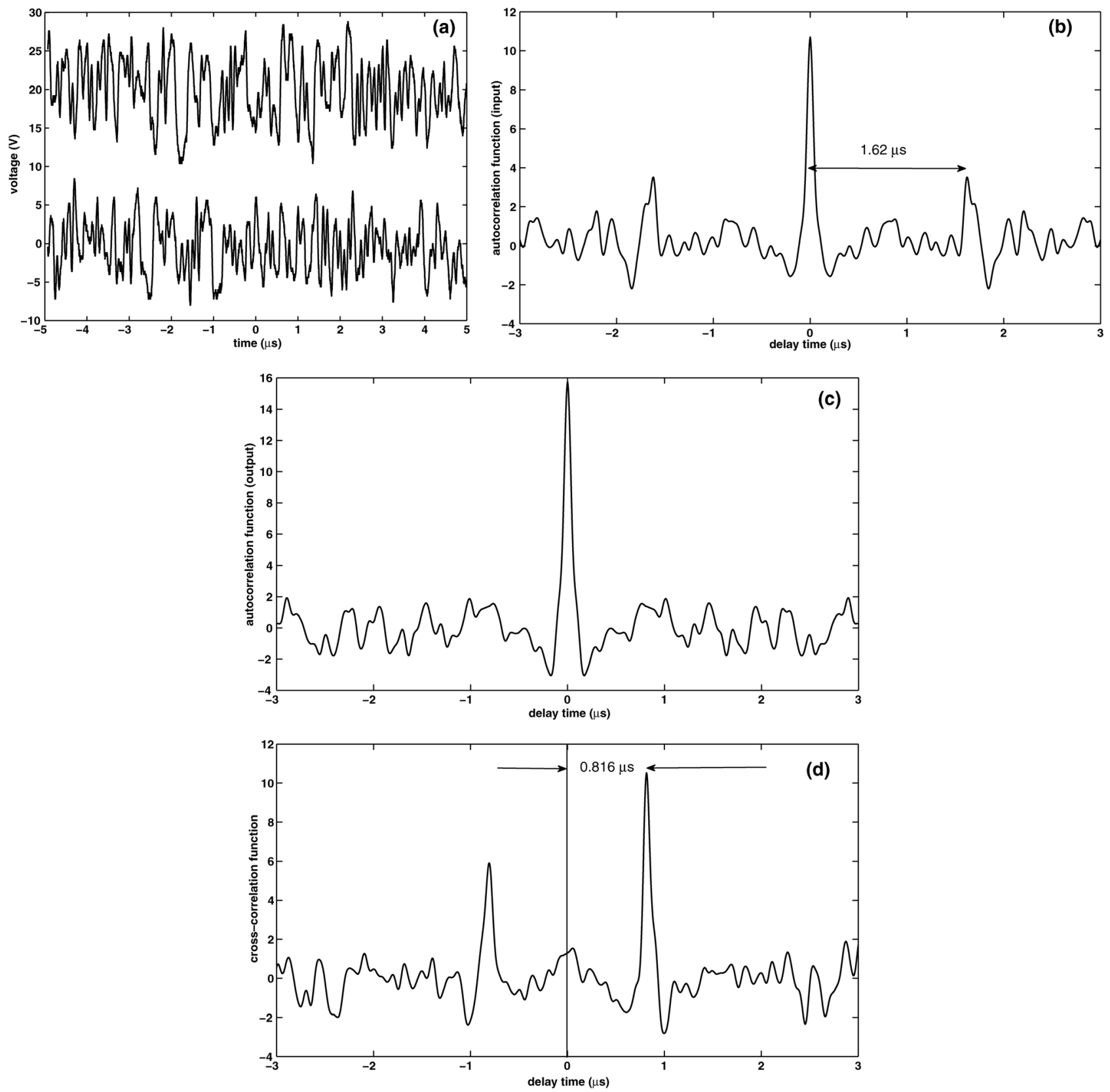

Fig. 3. Line response to a broadband noise. (a) Signals measured at the ends of the transmission line when numerically synthesized broadband noise is applied to the input. The signal at the output of the line has been displaced by $20 \mathrm{~V}$ to improve the visibility of the graph. The output of the line is open and the external conductor is grounded. (b) Autocorrelation function of the signal at the input of the line. There are two symmetrical peaks that correspond to twice the time of transit of electromagnetic pulses along the line. (c) Autocorrelation function of the signal at the output of the line. (d) Cross-correlation function of the signals. The two visible maxima correspond to $t=0.816 \mu \mathrm{s}$ and $t=-0.816 \mu \mathrm{s}$. The autocorrelation and cross-correlation functions are computed similarly to those in Fig. 2. 
existence of these two peaks reflects the fact that the signal at the input is a sum of the generator signal and the wave reflected at the end of the line. No correlations, other than the peak at $t=0$, are visible on the autocorrelation function of the output [see Fig. 3(c)].

The features of the autocorrelation functions at both ends of the line depend on the reflection coefficients at the ends. In the configuration that I have used the reflection coefficient at the output is $\Gamma_{L}=1$ and the reflection coefficient at the input is $\Gamma_{g}=-0.2$. $\left(\Gamma_{L, g}=\left(R_{L, g}-Z\right) /\left(R_{L, g}+Z\right)\right.$, where $\Gamma_{L, g}$ is the reflection coefficient, $R_{L}$ is the load resistance, $R_{g}$ is the internal resistance of the generator, and $Z$ is the characteristic impedance of the line.)

Figure $3(d)$ is a plot of the cross correlation function of the signals plotted in Fig. 3(a). The expected peaks at $\pm(0.816 \pm 0.004) \mu \mathrm{s}$ are observed. The peak at $-0.816 \mu \mathrm{s}$ is smaller because it corresponds to the disturbances reflected at the ends of the line. These peaks are very sharp, because they come from an almost white noise signal. The closer the original signal is to a white noise, the sharper the peaks. Other values of $R_{L}$ and $R_{g}$ lead to different amplitudes of the peaks in the autocorrelation and cross-correlation functions, but the characteristic times, $d / v$ and $2 d / v$, will remain unchanged.

\section{ACKNOWLEDGMENTS}

The author acknowledges fruitful discussions with Professors Rafael R. Boix, Francisco Medina, and Antonio Castellanos. The author is indebted to one of the referees for valuable comments. This work has been carried out with financial support from the Spanish Ministerio de Ciencia y Tecnología (MCYT) under Research Project No. FIS2006-03645 and Junta de Analucía under research projects EXC/2005/FQM421, P10-FQM-5735, and P09-FQM-4584.

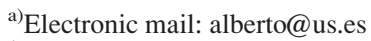

${ }^{1}$ P. Nakroshis, M. Amoroso, J. Legere, and C. Smith, "Measuring Boltzmann's constant using video microscopy of Brownian motion," Am. J. Phys. 71(6), 568-573 (2003).

${ }^{2}$ Y. Krafmakher, "Two student experiments on electrical fluctuations," Am. J. Phys. 63(10), 932-935 (1995).

${ }^{3}$ C. Beenakker and C. Schönenberger, "Quantum shot noise," Phys. Today 56(5), 37-42 (2003).

${ }^{4}$ N. Garnier and S. Ciliberto, "Nonequilibrium fluctuations in a resistor," Phys. Rev. E 71, 060101-1-4 (2005).

${ }^{5}$ B. Berne and R. Pecora, Dynamic Light Scattering (John Wiley \& Sons, New York, 1976).

${ }^{6}$ R. Snieder and K. Wapenaar, "Imaging with ambient noise," Phys. Today 63(9), 44-49 (2010).

${ }^{7}$ Y. Kraftmakher, "Correlation analysis with ScienceWorkshop," Am. J. Phys. 70(7), 694-697 (2002).

${ }^{8}$ G. H. Watson, "Transmission line excercises for the introductory physics laboratory,” Am. J. Phys. 63(5), 423-425 (1995).

${ }^{9}$ Se-yuen Mak, "Speed of radio waves along a coaxial transmission line," Am. J. Phys. 72(5), 671-675 (2004).

${ }^{10}$ I stored the acquired signal using the USB flash memory connection of the oscilloscope. Alternatively, the oscilloscope can be controlled directly from a computer via a USB connection. Depending on the software version and settings, the two channels may be acquired in sequence, not simultaneously, thus destroying the expected temporal correlation.

${ }^{11}$ See www.tti-arb.com. 